\title{
Clinical services for sleep disorders
}

\author{
Gregory Stores, Luci Wiggs
}

\begin{abstract}
Children's sleep disorders are common and often harmful to development and well being. The clinical services available to affected children and their families need to be improved. At present, professional interest and expertise in sleep disorders medicine is severely limited by the paucity of appropriate teaching and training. The work of a mainly tertiary sleep disorders clinic was reviewed, which showed that accurate diagnosis of a wide range of sleep disorders is possible, and that treatment needs can be specified. Although families appreciated such assessment, the outcome was unsatisfactory in many cases, often because treatment recommendations were not implemented by referrers. Reasons for this appear to include poor communication between referrers and families, and unavailability of treatment resources. A three tier system of service provision is proposed to improve this situation, which rests essentially on better professional training in the sleep disorders field.

(Arch Dis Child 1998;79:495-497)
\end{abstract}

Keywords: sleep disorders; health services

Children's sleep disorders are common; they are diverse in type, aetiology, and treatment requirements, and frequently serious in their effects on various aspects of development. Ample justification for such assertions is contained in the two recent comprehensive accounts of paediatric sleep disorders medicine by Ferber and Kryger ${ }^{1}$ and Dahl, ${ }^{2}$ and in the reviews by Stores $^{3}$ and Rosen. ${ }^{4}$ The main points worth emphasising are: (1) $\sim 25 \%$ of children, from infancy to adolescence, have sleep problems of major concern to them and their families; (2) the factors underlying such problems cover many physical and psychological possibilities; (3) the types of sleep disorder and varieties of treatment possibilities are much greater in number than conventional accounts in textbooks suggest; and (4) if these sleep problems are overlooked or mishandled, their effects on psychological and sometimes physical development can be serious and potentially long lasting. All these points apply to children in general; children in certain clinical groups, notably those with chronic physical illness, psychiatric disturbance, or learning disability, are at an even greater risk of sleep disorders and their consequences (Stores, unpublished data, 1998).

In spite of the compelling argument that sleep and its disorders are a very important aspect of child development and paediatric practice, the topic features very little in the teaching and training of doctors and other professionals including paediatricians, psychologists, and others involved in the care of children. The median time formally allocated to UK medical student teaching in sleep and its disorders was recently shown to be five minutes overall: five minutes in the paediatric and psychiatric parts of the course, and zero minutes in the general practice course. ${ }^{5}$ The situation in UK postgraduate medical training is not really known but anecdotally seems likely to be minimal, with only passing reference in the syllabus for training in paediatrics. ${ }^{6}$ The situation is similar in health visitor training apart from sporadic attempts in some localities. Surveys of undergraduate and clinical psychology courses in the UK show equally unimpressive teaching and training. ${ }^{78}$ This neglect appears to be universal, judging by surveys in the $\mathrm{USA}^{9}$ and Europe. ${ }^{10}$

Such services are not being provided Hard evidence about the provision of clinical services for children with sleep disorders and their families does not exist. Indeed, information about adult services is very sparse. In 1993, the British Sleep Society compiled a list of centres in the UK providing some type of sleep disorders clinic. Thirty seven centres provided information on the nature of their clinical service. Twenty four were essentially concerned with respiratory sleep disorders and only two of the total indicated a paediatric component. We know of no significant change since these figures were compiled.

Of course, many affected children are seen outside special centres by general practitioners, health visitors, paediatricians, child psychiatrists, and others for sleep problems but, although no systematically compiled information is available, it seems likely that very many children and adults with sleep disorders do not reach professional attention at all and, when they do, the provision is inadequate. Such help as is provided seems to be restricted largely to problems of settling and night waking in toddlers or nocturnal enuresis in older children, with little attention to the wide range of other types of sleep problem.

This limited awareness of sleep disorders is well illustrated by the case of obstructive sleep apnoea (OSA), the prevalence of which in childhood is estimated at $1 \% .{ }^{11}$ It would be surprising if anything like this number of children come to medical attention; it is thought that only $10 \%$ of adults with OSA are referred for assessment and treatment, in spite of growing awareness of the condition. In addition, recent studies of children with severe learning disability have demonstrated how relatively few 
Table 1 Sleep disorders diagnosed in the clinic series

\begin{tabular}{llc}
\hline Category of sleep disorder & Type of sleep disorder & $\begin{array}{c}\text { Number of } \\
\text { patients }^{*}\end{array}$ \\
\hline $\begin{array}{l}\text { Dyssomnias } \\
\text { Intrinsic sleep disorders }\end{array}$ & Narcolepsy & 7 \\
Extrinsic sleep disorders & Obstructive sleep apnoea & 1 \\
& Sleep onset association disorder & 11 \\
& Limit setting disorder & 7 \\
& Inadequate sleep hygiene & 1 \\
Circadian rhythm sleep disorders & Nocturnal eating and drinking syndrome & 1 \\
& Delayed sleep phase syndrome & 8 \\
Parasomnias & Advanced sleep phase syndrome & 2 \\
Arousal disorders & Irregular sleep/wake pattern & 2 \\
Sleep wake transition disorders & Sleepwalking & 10 \\
Associated with REM sleep & Sleep terrors & 9 \\
Other & Shythmic movement disorder & 6 \\
Sleep disorders arising from mental, & Neurological, or other medical disorders & 2 \\
Psychiatric disorders & Mood or anxiety disorders & 1 \\
Neurological disorders & Epilepsy & 1 \\
\hline
\end{tabular}

*Some patients had multiple disorders.

parents seek help or are provided with assistance for what are often very serious sleep disorders, probably because of a widespread lack of awareness of treatment possibilities. ${ }^{12}$

\section{Effective provision is possible (in principle)}

We have recently reviewed our children's outpatient sleep disorders service. This revealed a perceived widespread need for help with the assessment and management of such disorders, and that an accurate diagnosis, with specific treatment recommendations, is possible for most children (largely by means of informed clinical inquiry without the need in most cases for sophisticated technical investigations).

We looked at 78 children and adolescents referred consecutively to the clinic provided by the university department of psychiatry at the Park Hospital for Children in Oxford. Fifty nine per cent of the group were boys. The mean age was 9.6 years (range, 9 months to 17 years 8 months). Thirty two per cent were referred by child psychiatrists, $29 \%$ by paediatricians, and $26 \%$ by general practitioners, the remainder were referred by neurologists or psychologists. Fifty six per cent lived outside the Oxford area. Almost two thirds had coexisting medical conditions and almost half had psychiatric problems. Some degree of learning disability was evident in about a quarter, with school difficulties being prominent in almost two thirds.

The types of sleep problems described on referral were difficulties in settling to sleep or troublesome waking at night $(41 \%)$, episodes of disturbed behaviour associated with sleep $(36 \%)$, or excessive daytime sleepiness $(23 \%)$. The average overall duration of these sleep disorders was 5.2 years (range, 1 month to 17 years). The mainstay of the diagnostic process was a comprehensive clinical history (including a detailed sleep history) and assessment of the child's physical and psychological condition, the family's circumstances, and parental practices. A three to four week sleep diary record was often obtained and further special investigations arranged if necessary. Polysomno-
Table 2 Treatments and recommendations

\begin{tabular}{ll}
\hline Treatment & Percentage of total \\
\hline Behavioural intervention & 51 \\
Chronotherapy & 17 \\
Family work & 16 \\
Medication & 10 \\
Sleep hygiene advice only & 6 \\
\hline$\star$ Combinations common &
\end{tabular}

${ }^{\star}$ Combinations common.

graphy as an inpatient (sometimes with video monitoring) was considered appropriate in only $6 \%$ of cases.

By these means, it was possible to diagnose the specific sleep disorder underlying the sleep problem in $87 \%$ of cases. Failure to make a diagnosis was principally the result of parents not returning diary information or not providing details about family factors of possible relevance to the sleep problem, but difficulty in obtaining assessments elsewhere was often also a problem. Table 1 shows the range of sleep disorders identified, in accordance with international classification of sleep disorders criteria. $^{13}$

A wide variety of children's sleep disorders were recognisable for which referrers expressed a need for diagnosis and advice on treatment. That this need was shared by parents was reflected in the rarity with which families failed to keep their appointment at the clinic $(4.8 \%)$.

After diagnosis, treatment plans were formulated and implemented by the sleep clinic staff for some of the children living within the Oxford area and, in the remainder of children, treatment plans were recommended to referrers. Table 2 shows the range of treatments and recommendations. In addition to specific treatments, general advice was given on how to help the child to sleep well. This advice on "sleep hygiene" was mainly concerned with ensuring that the sleeping environment was conducive to sleep, encouraging regular sleeping routines, and avoiding influences likely to make it difficult to get to sleep and sleep soundly.

\section{Problems standing in the way of effective provision}

We were encouraged that almost nine out of 10 of this series of "difficult" cases were diagnosable, and that we could specify treatments required. However, the outcome was often disappointing.

We assessed the outcome for children in whom treatment had been implemented or recommended, collecting information from referrers, general practitioners, and families four months to five years (average, 2 years 3 months) after attendance at the clinic. Information from these sources, combined into an overall outcome measure, suggested that resolution or improvement of the sleep problem had occurred in only $45 \%$, with the situation unchanged or worse in $20 \%$. We could not judge the outcome in the remaining 35\% because clinical records contained no information on this point and informants did not respond to our inquiries. The factor most clearly associated with unsatisfactory outcome was referral from outside the immediate area, 
with recommendations having been made to referring agencies rather than implemented by the clinic's own staff.

The barriers to successful outcome were not related to the complexity of the sleep disorders diagnosed or the treatment recommended. Instead, the problems resulted mainly from poor communication between referrers and their families regarding treatment procedures, failure to appreciate the nature of the recommendations in spite of detailed descriptions of what was needed, or lack of suitably qualified or confident health professionals to implement them. These difficulties resulted in delays (sometimes prolonged) in providing treatment, confusion about how to proceed, and inevitable disappointment on the part of families. In spite of these difficulties, most families expressed their appreciation of the assessment process and their willingness to recommend it to other parents.

\section{Solutions to these problems}

The essential requirement for more adequate detection, assessment, and treatment of children's sleep problems is a substantial all round improvement in education and training concerning sleep and its disorders.

The starting point needs to be health education for parents, teachers, and (above a certain age) patients. The importance of adequate sleep for satisfactory functioning in many aspects of life needs to be appreciated more generally and ways of achieving this from an early age made known. Persistent sleeplessness in children (including those with a learning disability) should not be seen as inevitable, but as treatable and often preventable. Excessive daytime sleepiness should not be mistaken for laziness or disinterest, but as a condition (sometimes physical in origin) for which treatment is available. Likewise, episodic disturbances of behaviour associated with sleep (parasomnias) need a balanced, informed view taken of them, with further investigation where appropriate.

Increased professional interest and competence is also required. Shortcomings in teaching and training could be rectified by the introduction of a core curriculum for each professional group at undergraduate or postgraduate level. If carefully compiled and focused, this need not overload increasingly crowded programmes. In the case of medical students, sleep and sleep disorders illustrate well the general themes in medical education recommended recently by the General Medical Council-that is, their widespread impact; integration of basic medical science and clinical issues; the interspecialty and interdisciplinary nature of accurate diagnosis and effective management; and developmental aspects of the nature, presentation, management, and prognosis of sleep disorders from infancy to old age. ${ }^{14}$ In fact, these principles are appropriate for any course at whatever level.

The need in any sleep disorders service to integrate aspects of general paediatrics, paediatric neurology, respiratory medicine, develop- mental psychology, and child psychiatry requires a departure from the traditional separation between specialties and disciplines in teaching and training, and in the publication of clinical scientific work. We believe it unfortunate that articles on children's sleep disorders appear in a wide range of journals, each with a separate readership, and recommend some editorial means of combatting this separatism.

Ideally, with improvement in current education and training, a three tier system of provision would be possible for children's sleep disorders. Many sleep problems could be assessed and treated adequately at primary care level, especially the common settling and night waking problems in toddlers, for whom help is already provided by some health visitors, and nocturnal enuresis. More difficult diagnostic and treatment problems would require referral to paediatric services, preferably working closely with child psychiatrists. Where these measures had limited effect, or where the problem posed special diagnostic, assessment, or treatment difficulties, we recommend referral to a tertiary service (perhaps regional). In this last case, it would be essential that an identified professional in the child's own locality ensures that the process does not founder after the assessment stage, and that treatment needs are met without delay, with each child's progress monitored afterwards. Throughout the process, it would be essential to ensure that parents and professionals were working to the same agenda, with mutual understanding of the aims and the means of achieving them. In this way, effective help would be provided for the many children who need it.

1 Ferber R, Kryger MH. Principles and practice of sleep medicine in the child. Philadelphia: Saunders, 1995.

2 Dahl R, ed. Sleep disorders. Child Adolesc Psychiatr Clin North Am 1996;5:543-767.

3 Stores G. Practitioner review: assessment and treatment of sleep disorders in children and adolescents. $\mathcal{F}$ Child Psychol Psychiatry 1996;37:907-25.

4 Rosen CL. Sleep disorders in infancy, childhood and adolescence. Current Opinion in Pulmonary Medicine 1997; 3:449-55.

5 Stores G, Crawford C. Medical student education about sleep and its disorders. $\mathcal{F} R$ Coll Physicians Lond 1998;32: 149-53.

6 Royal College of Paediatrics and Child Health. Syllabus and training record for general professional and higher specialist training in paediatrics and child health. London: Royal College of Paediatrics and Child Health, 1996.

7 Wiggs L, Stores R. Sleep education in undergraduate Wiggs L, Stores R. Sleep education in undergraduate
psychology degree courses in the UK. Psychology Teaching Review 1996;5:40-6.

8 Stores R, Wiggs L. Sleep education in clinical psychology courses in the UK. Clin Psychol Forum 1998;119:14-18.

9 Rosen RC, Rosekind M, Rosevear C, Cole WE, Dement WC. Physician education in sleep and sleep disorders: a national survey of US medical schools. Sleep 1993;16:24954.

10 Salzarulo P. Workshop on education about sleep in Europe: chairman's summary. In: Horne JA, ed. Sleep 90, Proceedings of the tenth european congress on sleep research. Bochum: Pontnagel Press, 1990:475-8.

11 Carroll JL. Sleep-related upper airway obstruction in children and adolescents. Child Adolesc Psychiatr Clin North Am 1996:5:617-48.

12 Wiggs L, Stores G. Sleep problems in children with severe intellectual disabilities: what help is being provided? intellectual disabilities: what help is being provided? Fournal of

13 American Sleep Disorders Association. International classification of sleep disorders, revised. Diagnostic and coding manual. Rochester, Minnesota: American Sleep Disorders Association, 1997.

14 Education Committee of the General Medical Council. Tomorrow's doctors. Recommendations on undergraduate medical education. London: GMC, 1993. 